\title{
PRODUÇÃO DE CORDEIROS DA RAÇA SUFFOLK EM DOIS SISTEMAS DE MANEJO REPRODUTIVOS ${ }^{1}$
}

\author{
SUFFOLK LAMBS PRODUCTION IN TWO MATING SYSTEMS
}

\author{
Domingos Sanchez Roda ${ }^{2}$ Luiz Eduardo dos Santos ${ }^{3}$ Eduardo Antonio da Cunha ${ }^{2}$ \\ Mauro Sartori Bueno ${ }^{3}$ Ivani Pozar Otsuk ${ }^{4}$
}

RESUMO

Foram utilizadas 32 ovelhas Suffolk por dois anos em dois sistemas de monta, com acasalamentos a cada oito meses (alternativo) ou a cada doze meses (tradicional), visando a reduzir a estacionalidade da disponibilidade de animais para abate. Não foram observadas diferenças $(P>0,05)$ na fertilidade $e$ prolificidade dos animais, sendo no sistema alternativo, $66,7 \%$ e $110,4 \%$ e no tradicional 78,1\%; 103,1\% , obtendo-se 53 e 33 crias nascidas no período, respectivamente. Para a mortalidade das crias, observou-se, respectivamente, $28,3 \%$ e $6,1 \%(P<0,01)$. $O$ peso médio das crias ao nascerem foi de 3,46 e 4,45kg. $(P<0,05)$ e ao desmame (60 dias) foi de 18,73 e 18,86kg (P>0,05) $e$ o peso da ovelha à parição foi de 68,06 e 71,05kg (P>0,05), para o sistema alternativo e tradicional, respectivamente. Conclui-se que o sistema de monta alternativo possibilita a obtenção de maior número de cordeiros, sem prejuízo no desempenho ponderal das ovelhas e dos cordeiros, bem como uma melhor distribuição na produção de cordeiros para abate durante o ano.

Palavras-chave: cordeiros, fertilidade, manejo da reprodução, ovelha, prolificidade, Suffolk

\section{SUMMARY}

Thirty-two Suffolk ewes were utilized for two years in two different mating systems: a traditional (mating at every twelve months) and an alternative (at every eight months), aimed to reduce the seasonality of available animal to slaughter. The fertility and prolificity of ewes in the alternative system were 66.7 and $110.4 \%$ and in the traditional system 76.1 and $103.1 \%$. It was obtained 53 and 33 lambs in the period. Lamb mortality were 28.3 and $6.1 \%(P<0.01)$. Birth weight were 3.46 and $4.45 \mathrm{~kg}$ $(P<0.05)$; weaning weigth (60 days) were 18.73 and $18.86 \mathrm{~kg}$ $(P>0.05)$ and parturition ewe weight were 66.06 and $71.05 \mathrm{~kg}$ $(P>0.05)$ for alternative and traditional system, respectively.
Alternative mating system made possible to obtain larger number of lambs, without deleteriou effect to ewes and lambs performances, as well as a better distribution in the number of lamb for slaughter during the year.

Key words: ewes, fertility, lamb, prolificity, reproduction system, Suffolk.

\section{INTRODUÇÃO}

Os ovinos, notadamente das raças originárias de regiões temperadas, tendem a apresentar, nessas regiões, comportamento poliéstrico estacional (VIEIRA, 1967). Este comportamento tem determinado o sistema de manejo reprodutivo tradicionalmente utilizado pelos ovinocultores em nosso meio, com a realização do acasalamento das matrizes a cada 12 meses, normalmente no período entre dezembro a março. Isso determina a obtenção de um único ciclo reprodutivo por fêmea no ano, limitando o número de crias obtidas, e resultando na manutenção de um grande percentual de fêmeas vazias no plantel, durante uma parte significativa do ano. Além disso, essa concentração da atividade reprodutiva em uma época do ano dificulta o atendimento da demanda de mercado, por cordeiros para abate, no restante do período, representando um entrave à consolidação e ampliação do mercado consumidor.

Dados experimentais com as raças Merino Australiano, Ideal e Corriedale acasaladas a cada 12

\footnotetext{
${ }^{1}$ Projeto IZ-007/93 - Financiado pela FAPESP. SP. E-mail: ceam@izsp.br. Autor para correspondência.

${ }^{3}$ Pesquisador Científico do Instituto de Zootecnia - Centro de Nutrição e Alimentação Animal.

${ }^{4}$ Pesquisador Científico do Instituto de Zootecnia - Centro de Métodos Quantitativos.

${ }^{2}$ Pesquisador Científico do Instituto de Zootecnia, Centro de Etologia, Ambiência e Manejo, Caixa Postal 60, 13460-000, Nova Odessa, 
meses, em nosso meio, foram levantados através de trabalhos de PRUCOLI \& BACCARI Jr. (1967) e SILVA NETO et al. (1976), mostrando uma maior frequiência de animais em cio no período de dezembro a março, conforme a raça estudada. RODA et al. (1990), estudando aspectos reprodutivos em ovinos das raças Ideal e Corriedale acasalados em dezembro/janeiro ou abril/maio, verificaram que, embora não existam vantagens em se provocar a parição tardia, não foram observadas diferenças nas taxas de mortalidade, do nascimento ao desmame, nas duas estações do ano. Para a raça Ideal, houve um melhor desempenho nos animais provenientes da cobertura tardia. Esses dados evidenciaram ainda a possibilidade de realização de acasalamentos tardios, fora das épocas tradicionais, com essas raças.

Outras técnicas de manejo reprodutivo, diferentes da monta à cada 12 meses, foram comentadas por BASSON et al. (1969), MORE O'FERRALL (1977), NOTTER \& COPENHAVER (1980), DZAKUMA et al. (1982), FOGARTY et al. (1984), CAÑEQUE et al. (1989), URRUTIA MORALES et al. (1989) e RODA et al. (1993), que estudaram o acasalamento de ovelhas a cada 8 meses, como forma de permitir uma exploração mais intensa das matrizes. A intensificação da atividade reprodutiva das ovelhas, através de coberturas mais freqüentes, possibilitaria a obtenção de um maior número de cordeiros com o mesmo número de matrizes, além de diminuir a sazonalidade no nascimento de cordeiros. Todavia, o maior desgaste fisiológico das matrizes pode levar à diminuição nos índices de fertilidade, bem como maior mortalidade de crias devido a nascimentos em épocas menos propícias. LEWIS et al. (1996) avaliaram o efeito de um sistema de reprodução intensificado, para aumentar a produção de cordeiros, sobre a fertilidade de ovelhas Dorset e observaram que os animais acasalados em épocas atípicas, ou com menor intervalo entre-partos, mostraram menor fertilidade.

O objetivo deste estudo foi comparar o número total de cordeiros produzidos em um determinado período, em dois sistemas de acasalamento, um tradicional, com monta a cada 12 meses, e outro alternativo, com monta a cada oito meses, visando a reduzir a estacionalidade da disponibilidade de animais para abate.

\section{MATERIAL E MÉTODOS}

O experimento foi conduzido na Unidade de Ovinos, do Centro de Etologia, Ambiência e Manejo do Instituto de Zootecnia em Nova Odessa, SP, a uma latitude $22^{\circ} 42^{\prime}$ Sul e longitude $47^{\circ} 18^{\prime}$ Oeste, com precipitação pluviométrica anual média de $1.300 \mathrm{~mm}$ e temperatura média de $22,3^{\circ} \mathrm{C}$. Foram utilizadas 32 ovelhas da raça Suffolk, de segunda parição, que permaneceram sob pastejo rotativo, em área com capim aruana (Panicum maximum Jack cv aruana), com lotação variável de acordo com a disponibilidade de forragem, tendo livre acesso a abrigo e suplementação composta de sal iodado e mistura mineral (na proporção 2:1). As ovelhas receberam diariamente suplementação volumosa composta de capim Elefante (Pennisetum purpureum, Schun cv guaçu) no verão e silagem de milho, no inverno, acrescida de ração concentrada, constituída de milho e farelos de soja, algodão e trigo, com $16 \%$ de Proteína Bruta e $80 \%$ de Nutrientes Digestíveis Totais, fornecida a partir de 30 dias antes do acasalamento, durante o período de cobertura, no terço final da gestação e durante o aleitamento, na quantidade de $500 \mathrm{~g} /$ cabeça/dia. As crias receberam suplementação alimentar, do nascimento até o desmame, aos 60 dias de idade, na quantidade de 250 $\mathrm{g} / \mathrm{cabeça/dia.} \mathrm{Todo} \mathrm{o} \mathrm{rebanho} \mathrm{recebeu} \mathrm{tratamento}$ anti-helmíntico periodicamente, em função da existência de sintomatologia clínica de verminose e do resultado do exame de fezes (OPG).

Os animais foram divididos em dois grupos, sendo um acasalado a cada oito meses (sistema alternativo) e o outro a cada doze meses (sistema tradicional), e permaneceram no mesmo tratamento durante todo período de ensaio. No sistema alternativo, as ovelhas foram cobertas entre 01 de janeiro a 15 de fevereiro de 1994, 01 de setembro a 15 de outubro de 1994 e 01 de maio a 15 de junho de 1995. Os nascimentos ocorreram entre 28 de maio a 18 de julho de 1994, 30 de janeiro a 13 de março de 1995 e 03 de outubro a 17 de novembro de 1995 . No sistema tradicional, os acasalamentos ocorreram entre 01 de janeiro e 15 de fevereiro dos anos de 1994 e 1995. As parições ocorreram entre 30 de maio a 15 de julho de 1994 e 1995.

Visando a eliminar o efeito de reprodutor, foi feito rodízio, possibilitando que cada um dos carneiros do plantel pudesse atender os dois grupos de ovelhas alternadamente. A monta foi controlada, identificando-se a ovelha em cio através do uso de animais deferectomizados (rufiões). Toda ovelha que apresentou cio foi acasalada duas vezes, com intervalo de 12 horas, sendo posteriormente colocada junto com outro rufião, sendo novamente acasalada, como da primeira vez, caso viesse a apresentar novo cio. Cada estação de monta teve 45 dias de duração. Os rufiões foram colocados em contato com as ovelhas, mesmo aquelas em aleitamento (sistema alternativo), 15 dias antes do início de cada estação de monta, visando a induzir a ocorrência de cios. 
O efeito de cada sistema de acasalamento sobre a fertilidade e prolificidade das ovelhas, bem como sobre a mortalidade pré-desmame das crias, considerando-se o total do período experimental, foi estudado através do teste do qui-quadrado, considerando-se uma tabela $2 \times 2$, onde foi analisado, para fertilidade, o número total de parições ocorridas sobre o total de parições possíveis no período. Para a prolificidade, considerou-se o número total de cordeiros nascidos no período sobre o número total de parições possíveis no período. Para a mortalidade pré-desmame, foi considerado o número total de cordeiros mortos sobre o número total de cordeiros nascidos.

O efeito dos sistemas de acasalamento sobre o peso da ovelha à parição e peso da cria ao nascer e ao desmame (60 dias) foi estudado através de um delineamento inteiramente ao acaso, em esquema fatorial, conforme modelo: Yijm $=\mathrm{m}+\mathrm{Si}+$ $\mathrm{Tj}+\mathrm{Pm}+$ Eijm, onde Yijm $=$ peso do cordeiro ao nascer ou ao desmame ou peso da ovelha à parição, $\mathrm{m}=$ média populacional, $\mathrm{Si}=$ efeito do sexo da cria, $\mathrm{Tj}=$ efeito do tipo de nascimento, $\mathrm{Pm}=$ efeito do período entre acasalamento, Eijm $=$ erro experimental.

\section{RESULTADOS E DISCUSSÃO}

A Tabela 1 mostra que o percentual de fertilidade no período total foi menor para o sistema alternativo $(66,7 \times 78,1 \%)$, porém, quando analisado o número total de parições no período experimental, verificou-se que não houve diferença significativa $(\mathrm{P}>0,05)$ entre os sistemas $(32 \mathrm{X} 25$ parições). No sistema alternativo, apesar de se ter o mesmo número de matrizes, as chances de reprodução foram maiores, pois o maior número de estações de monta possibilitou, mesmo com menores valores percentuais de fertilidade, obter-se um maior número de parições no mesmo período, e com o mesmo número de matrizes. Esses valores estão próximos aos de RIBEIRO (1995), que obteve, em rebanhos controlados de raças de corte, em sistema reprodutivo tradicional, no Rio Grande do Sul, índices de $60 \%, 64 \%$ e $76 \%$, respectivamente, para as raças Hampshire Down, Suffolk e Ile de France. Verificou-se ainda que o menor índice de fertilidade ocorreu na cobertura de setembro $(43,8 \%)$, quando comparado aos índices médios de janeiro (56,3\% no sistema alternativo e $78,1 \%$ no tradicional) e de maio (100\%). LEWIS et al. (1996) também observaram menores índices de fertilidade em animais submetidos a acasalamentos fora do período tradicional, bem como naqueles com menor período entre partos. Todavia, os resultados obtidos no presente trabalho mostram a necessidade de se avaliar, de forma mais apurada, os fatores que determinaram esses valores mais baixos, notadamente para a monta de setembro, de forma a se tentar melhorar o índice de fertilidade no período total para o sistema de monta alternativo.

Situação se-

Tabela 1 - Datas das montas e índices de fertilidade e prolificidade de ovelhas Suffolk submetidas a dois sistemas de manejo reprodutivo (alternativo e tradicional) e mortalidade de cordeiros.

\begin{tabular}{|c|c|c|c|c|c|c|}
\hline \multirow{2}{*}{$\begin{array}{l}\text { Data da } \\
\text { Monta }\end{array}$} & \multicolumn{2}{|c|}{ Fertilidade } & \multicolumn{2}{|c|}{ Prolificidade } & \multicolumn{2}{|c|}{ Mortalidade } \\
\hline & $\begin{array}{l}\text { Alternativo } \\
8 \times 8 \text { meses }\end{array}$ & $\begin{array}{l}\text { Tradicional } \\
12 \times 12 \text { meses }\end{array}$ & $\begin{array}{l}\text { Alternativo } \\
8 \times 8 \text { meses }\end{array}$ & $\begin{array}{l}\text { Tradicional } \\
12 \times 12 \text { meses }\end{array}$ & $\begin{array}{l}\text { Alternativo } \\
8 \times 8 \text { meses }\end{array}$ & $\begin{array}{l}\text { Tradicional } \\
12 \times 12 \text { meses }\end{array}$ \\
\hline $01 / 94$ & $09 / 16$ & $11 / 16$ & $12 / 16$ & $11 / 16$ & $03 / 12$ & $01 / 11$ \\
\hline 09/94 & $07 / 16$ & - & $13 / 16$ & - & $03 / 13$ & - \\
\hline $01 / 95$ & - & $14 / 16$ & - & $22 / 16$ & - & $01 / 22$ \\
\hline $05 / 95$ & $16 / 16$ & - & $28 / 16$ & - & $09 / 28$ & - \\
\hline Totais & $\begin{array}{c}32 / 48 \\
(66,7 \%)\end{array}$ & $\begin{array}{c}25 / 32 \\
(78,1 \%)\end{array}$ & $\begin{array}{c}53 / 48 \\
(110,4 \%)\end{array}$ & $\begin{array}{c}33 / 32 \\
(103,1 \%)\end{array}$ & $\begin{array}{c}15 / 53 \\
(28,3 \%)\end{array}$ & $\begin{array}{l}02 / 33 \\
(6.1 \%)\end{array}$ \\
\hline$\chi^{2}$ & \multicolumn{2}{|c|}{$1,23^{\mathrm{ns}}$} & \multicolumn{2}{|c|}{$1,00^{\mathrm{ns}}$} & \multicolumn{2}{|c|}{$6,29^{* *}$} \\
\hline
\end{tabular}

Fertilidade $=n^{\circ}$ total de parições ocorridas no período $/ \mathrm{n}^{\circ}$ total de parições possíveis. Prolificidade $=\mathrm{n}^{\circ}$ total de cordeiros nascidos no período $/ \mathrm{n}^{\circ}$ total de parições possíveis. Mortalidade pré-desmame $=\mathrm{n}^{\circ}$ total de cordeiros mortos $/ \mathrm{n}^{\circ}$ total de cordeiros nascidos no período. $\mathrm{ns}=(\mathrm{P}>0,05), * *=(\mathrm{P}<0,01)$ melhante foi verificada para a prolificidade total nesse período, tanto em valores percentuais $\quad(110,4 \quad \mathrm{x}$ $103,1 \%)$, como em valores reais, onde $\mathrm{o}$ número de crias obtidas no sistema alternativo não diferiu significativamente $(\mathrm{P}>0,05)$ nos dois sistemas $(53 \times 33$ cordeiros).

Já para a
mortalidade desmame, o número total de óbitos verificado no sistema de monta alternativo foi significativamente superior $(\mathrm{P}<0,01)$ ao observado no sistema de monta tradicional $(15 \quad \mathrm{X} \quad 2$ óbitos), resultando em 
percentuais de $28,3 \%$, no sistema alternativo e $6,1 \%$, no sistema tradicional. $\mathrm{O}$ valor verificado no sistema de monta alternativo é superior tanto aos observados por RODA et al. (1990), para ovinos das raças Ideal e Corriedale provenientes de estação de monta tradicional, como aos verificados por RODA et al. (1993), para ovinos das raças Ideal, Corriedale e Suffolk, da ordem de $12 ; 16,5$ e $11,6 \%$, respectivamente, para as crias nascidas das estações de monta de janeiro, setembro e maio. Já o valor verificado no sistema tradicional é menor que os $12 \%$ observados pelos mesmos autores para a monta em janeiro. A maior mortalidade observada no sistema alternativo pode ser devido, não só ao menor peso ao nascer das crias desse sistema (Tabela 2), como também em função de fatores ambientais, que resultaram em elevada taxa de mortalidade das crias oriundas da cobertura de maio (parição em outubro/novembro) no sistema de monta alternativo.

O peso vivo médio das crias ao nascerem foi significativamente menor $(\mathrm{P}<0,01)$ no sistema alternativo, evidenciando um efeito negativo da intensificação da atividade reprodutiva da ovelha sobre esse parâmetro, em comparação ao sistema de monta tradicional. Todavia, não se observaram diferenças significativas $(\mathrm{P}>0,05)$ entre os dois sistemas no peso à desmama, mostrando que se a intensificação da atividade reprodutiva prejudicou o crescimento do feto durante a gestação, não houve prejuízo à produção de leite das ovelhas, nem ao ritmo de crescimento das crias. Não foi observado efeito significativo do sistema de manejo reprodutivo sobre o peso vivo das ovelhas à parição, o que pode explicar o fato das crias resultantes do sistema de monta a cada 8 meses terem apresentado desempenho equivalente ao das crias resultantes do sistema de monta à cada 12 meses.

O peso vivo médio das crias de parto simples ao desmame foi significativamente maior $(\mathrm{P}<0,01)$ que o das crias de parto múltiplo, sendo que os partos múltiplos foram provenientes de ovelhas com maior peso à parição que os partos simples $(\mathrm{P}<0,05)$. Os valores obtidos neste trabalho para o peso vivo médio de crias, ao nascer e ao desmame, aproximam-se ou superam aqueles obtidos por RODA et al. (1981, 1983 e 1990), em qualquer dos sistemas de acasalamento, evidenciando que o manejo reprodutivo alternativo não prejudicou o desempenho ponderal das crias.

\section{CONCLUSÕES}

O sistema de monta alternativo possibilita a obtenção de maior número de cordeiros, sem prejuízo ao desempenho ponderal das ovelhas e dos cordeiros, assim como uma melhor distribuição na produção de cordeiros para abate durante o ano.

\section{REFERÊNCIAS BIBLIOGRÁFICAS}

BASSON, W.D., VAN NIEKERK, B.D.H., MULDER, A.M., et $a l$. The produtive and reproductive potential of three sheep breed mated at 8-monthly intervals under intensive feeding conditions. Proceeding of South African in Animal Production., Pretoria, v. 8, p. 149-154, 1969.

CAÑEQUE, V., HUILDOBRO, F.R., DOLZ, J.F. et al. Produccion de carne de cordero. Madrid: Ministério de Agricultura Pesca y Alimentaccion, 1989, 520 p.

DZAKUMA, J.M., STRIZKE, D.J., WHITEMAN, J.V. Fertility and prolificacy of crossbred ewes under two cycles of accelerated lambing. Journal of Animal Science., Albany, v. 54 , n. 2 , p. $213-220,1982$

FOGARTY, N.M., DICKERSON, G.E., YOUNG, L.B. Lamb production and its components in pure breeds and composite lines. I Seasonal and other environmental effects. Journal of Animal Science, Albany, v. 58, n. 2, p. 285-300, 1984.

LEWIS, R.M., NOTTER, D.R., HOGUE, D.E. et al. Ewe fertility in the STAR accelerated lambing system. Journal of Animal Science, Albany, v. 74, n. 7, p. 1511-1522, 1996.

MORE O'FERRALL, G.J. Reproductive performance of Finndorset ewes on an 8-mo lambing system. Animal Production. London, v. 24, n. 1, p. 129. 1977.

NOTTER, D.R., COPENHAVER, J.S. Performance of Finnish Landrace crossbred ewes under accelerated lambing. I Fertility, prolificacy and ewe productivity. Journal of Animal Science, Albany, v. 51, n. 5, p. 1033-42, 1980. 
PRUCOLI, J.O., BACCARI Jr., F. Estudos sobre estação de monta em ovinos no Estado de São Paulo. Boletim da Indústria Animal, São Paulo, SP., n. 24, p. 75-79, 1967.

RIBEIRO, L.A.O. Perdas reprodutivas em ovinos no Rio Grande do Sul: Causas e Soluções. In: SIMPÓSIO PAULISTA DE OVINOCUlturA, 4, 1995, Campinas. Anais... Campinas: CATI, 1995, p. 113-124.

RODA, D.S., SANTOS, L.E., CAMPOS, B.E.S. et al. Crescimento ponderaral de ovinos das raças Suffolk e Ideal do nascimento ao desmame. Boletim da Indústria Animal, Nova Odessa, SP, v. 38, n. 1, p. 31-43, jan./jun., 1981.

RODA, D.S., SANTOS, L.E., OLIVEIRA, A.A.D. $\boldsymbol{e} \boldsymbol{t} \boldsymbol{a l}$. Crescimento ponderal de cordeiros deslanados, Suffolk e cruzados deslanados $\mathrm{x}$ Suffolk. Boletim da Indústria Animal, Nova Odessa, SP, v. 40, n. 1, p. 25-30. jan./jun., 1983.

RODA, D.S., SANTOS, L.E., DUPAS W., et al.. Avaliação do peso ao nascer e ao desmame e mortalidade pré-desmame em cordeiros Santa Inês, Suffolk e cruzados Suffolk. Boletim da
Indústria Animal, Nova Odessa, SP, v. 47, n. 2, p. 153-7. jul./dez., 1990.

RODA, D.S., SANTOS, L.E., CUNHA, E.A., et al.. Desempenho de ovinos Ideal e Corriedale em sistema de acasalamento a cada oito meses. Boletim da Indústria Animal, Nova Odessa, SP, v. 50, n. 12, p. 49-54, jan./jun., 1993.

SILVA NETO, B.C., FERRARI, O., TOLEDO. J.R., et al. Estabelecimento da estação de cobertura para ovinos Corriedale no Sul do Estado de São Paulo. Boletim da Indústria Animal, São Paulo, SP, v. 33, n. 1, p. 145-152, 1976.

URRUTIA MORALES, J., MARTINEZ ROJA, L., GARCIA FIGUEROA, F.S., et al.. Caracteristicas reprodutivas de ovejas de la raca Rambouillet en Mexico. 2. Empradres cada 8 meses. Tecnica Pecuaria en Mexico. Mexico, v. 27, n. 2, p. 71-83, 1989.

VIEIRA, G.V.N. Produção de Ovinos. São Paulo: Melhoramento, 1967, 479 p.

Ciência Rural, v. 29, n. 1, 1999. 